\title{
Control of Schottky and ohmic interfaces by unpinning Fermi level
}

\author{
S. Hara * , T. Teraji ${ }^{1}$, H. Okushi, K. Kajimura ${ }^{1}$ \\ Electrotechnical Laboratory, 1-1-4 Umezono, Tsukuba, Ibaraki 305, Japan
}

\begin{abstract}
We propose the first systematical method to control Schottky barrier heights of metal/semiconductor interfaces by controlling the density of interface electronic states and the number of charges in the states. The density of interface states is controlled by changing the density of surface electronic states, which is controlled by surface hydrogenation and flattening the surface atomically. We apply establishing hydrogen termination techniques using a chemical solution, $\mathrm{pH}$ controlled buffered HF or hot water. Also, slow oxidation by oxygen gas was used to flatten resultant semiconductor surfaces. The density of interface charges is changeable by controlling a metal work function. When the density of surface states is reduced enough to unpin the Fermi level, the barrier height is determined simply by the difference between the work function of a metal $\phi_{\mathrm{m}}$ and the flat-band semiconductor $\phi_{\mathrm{s}}^{\mathrm{FB}}$. In such an interface with the low density of interface states, an ohmic contact with a zero barrier height is formed when we select a metal with $\phi_{\mathrm{m}}<\phi_{\mathrm{s}}^{\mathrm{FB}}$. We have already demonstrated controlling Schottky and ohmic properties by changing the pinning degree on silicon carbide (0001) surfaces. Further, on an atomically-flat $\mathrm{Si}(111)$ surface with monohydride termination, we have observed the lowering of an $\mathrm{Al}$ barrier height. Moreover, we found the recovery of an ohmic property after $\mathrm{TiC}$ formation at $\mathrm{Ti} / 6 \mathrm{H}-\mathrm{SiC}$ interface at $700^{\circ} \mathrm{C}$ whereas conventional 5\% HF rinsed Schottky Ti/6H-SiC interfaces still have Schottky properties after TiC formation.
\end{abstract}

Keywords: Fermi level pinning; Ohmic contact; Schottky contact; Monohydride; Interface states

\section{Introduction}

Ohmic contacts as non-rectifying electrodes and/or Schottky contacts as rectifying electrodes have been used in every discrete and integrated electronic devices since a diode was invented over half a century ago. Uncontrollability of Schottky barrier heights, however, restricts Schottky contacts within limited applications to only high speed de-

\footnotetext{
* Corresponding author. Tel.: + 81-298-585115; fax: + 58-298585419; e-mail: shara@etl.go.jp.

${ }^{1}$ Also with Faculty of Materials Science, University of Tsukuba, 1-1-1 Tennodai, Tsukuba, Ibaraki 305, Japan.
}

vices such as Schottky diodes or metal-semiconductor field-effect transistors (MESFET); Rectifying role is usually made by $\mathrm{p}-\mathrm{n}$ junctions. Also, the uncontrollability and inevitable formation of a Schottky barrier in every metal-semiconductor interface results in difficulty in the formation of an ohmic contact. In order to salve it, ohmic contacts are formed by leaking currents through the barriers instead of lowering the barrier heights. The leaky Schottky barriers have complex mechanisms on current transports because of complex interfacial atomic and electronic structures caused by interfacial reactions depending on experimental conditions. In practical devices, ohmic contacts by leaky Schottky bar- 
rier formation are fabricated by high doping of impurities so as to tunnel electron current through the barrier whose width is thinned. As a general trend, a Schottky barrier has been formed for an ideal abrupt interface, whereas an ohmic contact has been formed by deteriorating the interface abruptness or by lowering the crystallinity of the semiconductor. Conventional techniques to form Schottky and ohmic contacts are described in the text of Rhoderick [1].

Overcoming the uncontrollability will provide us wide applications of Schottky barriers as a stable function in devices and also ideal ohmic contacts with zero barrier height, which release us from difficulty in formation of the conventional ohmic contacts. The uncontrollability is mainly due to an interfacial Fermi-level pinning phenomenon which automatically determines the barrier height. Therefore, it is quite important to know the mechanism(s) of the pinning phenomenon and to develop how to unpin.

It is well-known that the Schottky barrier height $\phi_{\mathrm{bn}}$ is generally expressed as follows,

$\phi_{\mathrm{bn}}=S\left(\phi_{\mathrm{m}}-\chi_{\mathrm{s}}\right)+C$,

where we define the slope parameter $S$ as $\partial \phi_{\mathrm{bn}} / \partial \phi_{\mathrm{m}}$. $S$ has a range of $0 \leq S \leq 1$. The situation with $S=1$ is called the Schottky limit, where the Fermi level is free from pinning. When $S$ is $0, \phi_{\mathrm{bn}}$ in Eq. (1) becomes constant, and is independent of the metal work function $\phi_{\mathrm{m}}$. This situation of the strongest pinning is the Bardeen limit. Since in practical interfaces $S$ has values between the Schottky limit and Bardeen limit, we can control the barrier height to some extent by changing a metal. For instance, the maximum and minimum barrier heights for silicide/n-Si interfaces are around $0.5 \mathrm{eV}$ to $0.9 \mathrm{eV}$ and thereby the maximum width of controlling the barrier height is around $0.4 \mathrm{eV}$ [2], indicating that no ohmic interface is formed without high doping. So far, there existed no technique to control the pinning degrees, the Schottky barriers were out of control. As a result, each semiconductor has each fixed pinning degree.

In this report, we propose the first concept to unify the Schottky and ohmic formations by achieving the Schottky limit, where we utilize up-to-date chemical treatments to control surface termination. We also demonstrate barrier height controls and ohmic formations using $6 \mathrm{H}-\mathrm{SiC}(0001)$ and the
Si(111) substrates. Further, we demonstrate the thermal stability of the interfaces with Schottky limit.

\section{Unifying Schottky and ohmic formations by controlling the Fermi level pinning}

It is known that the pinning rate is dominated by the density of interface states $D_{\text {it }}$. The relation is $S=\epsilon_{\mathrm{i}} /\left(\epsilon_{\mathrm{i}}+q \delta D_{\mathrm{it}}\right)$, where $\epsilon_{\mathrm{i}}$ is the permittivity of the interfacial layer and $\delta$ its thickness [2]. Therefore, we can control the degree of the Fermi level pinning by changing $D_{\mathrm{it}}$. In terms of lowering $D_{\mathrm{it}}$, in-situ cleaning of a semiconductor surface in ultrahigh vacuum is not effective because of reconstruction induced surface pinning [3]. This leads to pinning at resultant interfaces. So far, there is a significant report aiming control of $S$ or $D_{\text {it }}$ using ex-situ chemical treatment. Fan et al. reported that $S$ of GaAs increases from 0.14 for conventional metal/GaAs interfaces to 0.53 by the sulfur termination of the surface [4]. The suggestive phenomenon in this technique is the retrieval of the controllability of $S$ by controlling surface termination. If a semiconductor surface is a completely terminated one without no dangling bonds, it should endure oxidation and contamination, resulting in an ideal interface with low $D_{\text {it }}$.

Recent techniques using chemical solutions to terminate semiconductor surfaces progress to reduce the density of surface states and make their surfaces flat atomically. Such an ideal surface should result in forming an interface with a low $D_{\text {it }}$. We utilize two chemical solutions, $\mathrm{pH}$ modified buffered HF or hot water to suppress $D_{\mathrm{it}}$. $\mathrm{pH}$ modified buffered HF has been found to terminate the $\mathrm{Si}(111)$ surface only by monohydrides [5]. Hot water also has the same effect [6]. In a semiconductor surface layer with a low crystallinity which becomes the origin of high $D_{\text {it }}$, removing the surface layer by an oxidation followed by a chemical etching is effective before the chemical termination. Also, slow oxidation is known to form an atomically-flat interface by enhancing a lateral oxidation speed relatively [7]. Atomically-flat surface is obtained by removing the oxide layer. This surface has a lower density of surface states.

Further, an actual barrier height is determined by the number of interface charges $Q_{\mathrm{it}}$ in $D_{\mathrm{it}} \cdot Q_{\mathrm{it}}$ is 
changeable if the metal work function $\phi_{\mathrm{m}}$ is changed because the change in Fermi levels between the metal and the semiconductor yields a charge transfer. When $D_{\mathrm{it}} \rightarrow 0$, the interface is in the Schottky limit. In the limit with zero $D_{\text {it }}$, the ohmic property is obtained when $\phi_{\mathrm{m}}$ is smaller than $\chi_{\mathrm{s}}$ because $C$ in Eq. (1) is generally small. Since this ohmic interface has no Schottky barrier, the contact resistivity $\rho_{\mathrm{c}}$ is zero, independent of the donor concentration $N_{\mathrm{D}}$. In contrast, in a common ohmic contact, there exists a large $D_{\text {it }}$ resulting in a pinned Schottky barrier formation restricting the current conductivity. In such a contact, the minimum of $\rho_{\mathrm{c}}$ is limited by the Schottky barrier width determined by $N_{\mathrm{D}}$ because the current flows through the Schottky barrier by tunneling.

\section{Demonstration of controlling barrier heights}

Unintentionally doped n-type $6 \mathrm{H}-\mathrm{SiC}(0001)$ bulk substrates grown by modified Lely method and nitrogen doped n-type 6H-SiC (0001) epitaxial layers were used. Si-faces of the $\mathrm{SiC}$ samples were used for all depositions and characterizations. The carrier concentrations $N_{\mathrm{D}}$ determined by capacitance versus voltage $(C-V)$ measurements were $\sim 2 \times 10^{17} \mathrm{~cm}^{-3}$ for the bulk substrates and $\sim 5 \times 10^{17} \mathrm{~cm}^{-3}$ for the epitaxial layers. Some of the samples were oxidized in a quartz tube furnace. The thickness of oxidized layers was about $3 \mathrm{~nm}$. The oxidized layers were etched by dipping in 5\% HF solution. Some of the samples with oxidation/etching treatment were dipped in boiling water or in $\mathrm{pH}$-modified buffered HF for $10 \mathrm{~min}$. Finally, the samples were rinsed in deionized water. Titanium, molybdenum, and nickel were deposited with an electron beam evaporator to form contact electrodes. During the depositions, the sample temperatures were kept below $100^{\circ} \mathrm{C}$. No annealing was carried out for all electrodes. Magnesium ohmic back contacts were deposited at room temperature. Most significant experimental technique here is to avoid a procedure of back ohmic formation before forward electrode formation. Otherwise, in the following cleaning procedures the cleaning surfaces have effects of annealing history and/or contamination by the back metal or its cap wax. Actu-

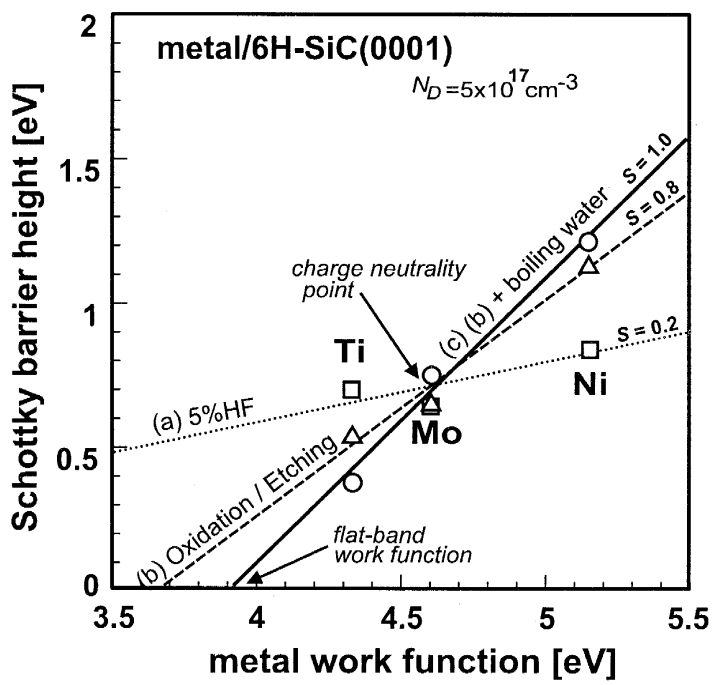

Fig. 1. Schottky barrier heights as a function of metal work functions. No treatment (short dashed line), oxidation followed by HF etching treatment (long dashed line), and dipping treatment in boiling water (straight line) are indicated.

ally, we have observed Ni Schottky contacts independent of the surface treatments when we form back ohmic contacts before Schottky electrodes [8].

Fig. 1 shows $\phi_{b n}$ as a function of $\phi_{\mathrm{m}}$ for the three treatments. Conventional treatment (a) has a slope parameter $S$ of 0.2 . After treatment (b) of oxidation followed by etching, $S$ rises to 0.8 . Treatment (c) using boiling water generates 1.0 , which is in the pinning-free Schottky limit. The Fermi level is being released from pinning in order with proceeding the treatments from (a) to (c).

Ni contacts in all treatments show Schottky properties and $\phi_{\mathrm{bn}}$ increases with proceeding the treatments from (a) to (c). In the Ti contacts $\phi_{\text {bn }}$ decreases with proceeding the treatments. $\phi_{\mathrm{bn}}$ of the Ti contact by treatment (c) is a calculated value from $\rho_{\mathrm{C}}$ of $6 \times 10^{-3} \Omega \mathrm{cm}^{2}$. In the Mo contacts, $\phi_{\mathrm{bn}}$ for all treatments are almost the same. A cross point of the three lines near the Mo plots, indicating $0.70 \mathrm{eV}$ of $\phi_{\mathrm{bn}}$, is the pinning position of the Fermi level because it is independent of the surface treatments. This position is called a charge neutrality level. The corresponding $\phi_{\mathrm{m}}$ at the charge neutrality level is around $4.6 \mathrm{eV}$ in the figure. The extrapolated work function at zero Schottky barrier is $3.9 \mathrm{eV}$ along the 
line of $S=1$. The work function is that of flat-band. Since $S$ is constant for each treatment, $\phi_{\mathrm{bn}}$ is linear for the change in $\phi_{\mathrm{m}}$. In other words, $S$ has no lowering behavior near the charge neutrality point at least in the metal $/ 6 \mathrm{H}-\mathrm{SiC}(0001)$ system.

When the Fermi level is at the charge neutrality point, the interface becomes electrically neutral. The amount of the band bending by the pinning $0.70 \mathrm{eV}$ is preserved after the deposition of a metal with the work function of $4.6 \mathrm{eV}$. Also on the pinning-free surface, the metal with that of $4.6 \mathrm{eV}$ also forms $\phi_{\mathrm{bn}}$ of $0.70 \mathrm{eV}$ because a difference of work functions between $\mathrm{SiC}$ and the metal is $0.7 \mathrm{eV}(=4.6-3.9)$. As the result, the metal at the charge neutrality point has the constant barrier height independent of the surface treatments by which the density of interface states widely varies.

We can estimate the electron affinity $\chi_{\mathrm{s}}$ using the observed flat-band work function $3.9 \mathrm{eV}$. Since the n-type dopant level by nitrogen is at $0.085 \mathrm{eV}$ below the level of conduction band minimum [9], $\chi_{\mathrm{s}}$ is estimated at around $3.8 \mathrm{eV}$. This is a new way to estimate $\chi_{\mathrm{s}}$.

Schottky and ohmic contacts in our experiments are formed on the lightly doped substrates $\left(N_{\mathrm{D}} \sim 10^{17}\right.$ $\left.\mathrm{cm}^{-3}\right)$. In such an interface with a wide depletion layer, a tunneling current is negligible. The dominant current transport is thermionic emission. Therefore, the ohmic currents in our experiments are generated by current flows over barriers with the low heights. Further, it should be noted that the ohmic contacts are formed without post-annealing.

We demonstrate the proposed technique also on $\mathrm{Si}(111)$ surfaces. According to the classical prediction of $S$ by Kurtin et al. in 1969 [10], $S$ of $\mathrm{Si}$ is around strongly pinned 0.02 , where we divide their value 0.05 by 2.27 to translate the metal parameter from the metal electronegativity to $\phi_{\mathrm{m}}$. In such an old period, the cleaning technique of semiconductor surfaces was not sophisticated well, resulting in the strong pinning. So, it is valuable to investigate $S$ again using the present advanced techniques of surface cleaning. The hardness of $\mathrm{Si}$, however, is much lower than SiC. The etching of the Si surface should be more careful. Otherwise, the resultant surface becomes rougher than that before the etching. So, we utilizes sophisticated procedures of cleaning to flatten the surface for $\mathrm{Si}(111)$.

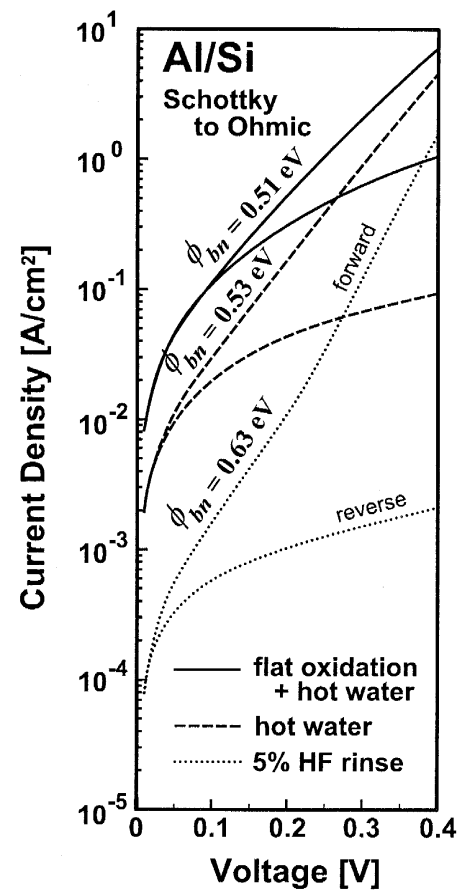

Fig. 2. Schottky barrier heights of $\mathrm{Al} / \mathrm{Si}(111)$ interfaces. The surface treatments for dotted, dashed, and solid curves are 5\% HF rinse, hot water immersion, and flat oxidation followed by hot water immersion.

Phosphorus-doped n-type $\mathrm{Si}(111)$ substrates with a carrier concentration of $7 \times 10^{16} \mathrm{~cm}^{-3}$ were used. The mis-oriented angle was $0.44^{\circ}$ toward $\langle 1 \overline{1} 0\rangle$. All samples were degreased followed by $5 \% \mathrm{HF}$ rinse for $60 \mathrm{~s}$. A part of the samples were metallized by aluminum to make electrodes using the electron beam evaporator. Some of the other samples were metallized after immersion in pure water at $90^{\circ} \mathrm{C}$. The rest samples just after the first 5\% HF rinse were oxidized at $1100^{\circ} \mathrm{C}$ in the quartz tube furnace. The oxide thickness was $200 \mathrm{~nm}$. The oxide layers were removed by $5 \% \mathrm{HF}$ rinse. The samples were cleaned using the procedure of RCA clean [11]. After the cleaning, they were oxidized again at $300^{\circ} \mathrm{C}$ followed by $800^{\circ} \mathrm{C}$ to flatten the $\mathrm{Si}$ surfaces. The oxides were removed by $5 \% \mathrm{HF}$ rinse. After that, RCA clean was performed again. The samples were also immersed in the hot water. Al was deposited after the procedure. Fig. 2 shows the $I-V$ characteristics of the samples with the three procedures, the $5 \% \mathrm{HF}$ 
rinse, the hot water immersion, and the hot water immersion after the flat oxidation. The barrier height is decreasing with proceeding the procedure from the simple 5\% HF through the hot water immersion to terminate the surface by monohydride to the procedure including the flat oxidation. Note that conventional oxidation at around $1000^{\circ} \mathrm{C}$ had no effect to decrease the barrier height. Also, a rectifying property, that is, the current difference between forward and reverse bias is disappearing. The samples with the flat oxidation followed by hot water immersion have the ohmic-like property because the current difference is within one order of magnitude. The remaining Schottky barrier of $0.51 \mathrm{eV}$ for the last procedure is an obstacle to lower the contact resistivity.

If we know the electron affinity $\chi_{\mathrm{s}}$ of $\mathrm{Si}$, we can estimate an ideal barrier height for $\mathrm{Al}$ in the pinning-free condition. $\chi_{\mathrm{s}}$, however, is a controversial parameter because it has no direct way to measure. If we use the value of $5.15 \mathrm{eV}$ as the ionization energy [12], the estimated $\chi_{\mathrm{s}}$ by subtracting the band gap width $1.12 \mathrm{eV}$ is $4.03 \mathrm{eV}$. By adding $0.16 \mathrm{eV}$ between Fermi level and conduction band minimum (for the carrier concentration of $7 \times 10^{16} \mathrm{~cm}^{-3}$ ) to $4.03 \mathrm{eV}, \phi_{\mathrm{n}-\mathrm{Si}}$ of the flat-band is calculated to be $4.19 \mathrm{eV}$. The estimated ideal Schottky barrier in the pinning-free condition is $0.09 \mathrm{eV}$ using the value of $4.28 \mathrm{eV}$ for $\phi_{\mathrm{Al}}$ [13]. This is much smaller than our observed $0.51 \mathrm{eV}$. This suggests that the surface with the flat oxidation followed by the hot water immersion still has an enough density of interface states to pin. A further advanced technique is required to lower the barrier height. Even in this unsatisfactory situation, it is surprising that the ohmic property is obtained by flattening the surface atomically and increasing the density of stable monohydride, which is an way completely contradictory to the conventional techniques to form ohmic contacts.

\section{Thermal stability of pinning-controlled inter- faces}

Significant concern about the pinning-controlled interfaces is thermal stability of the interfaces with the ideal low densities of interface states. Is initial electronic ideality at an interface preserved after

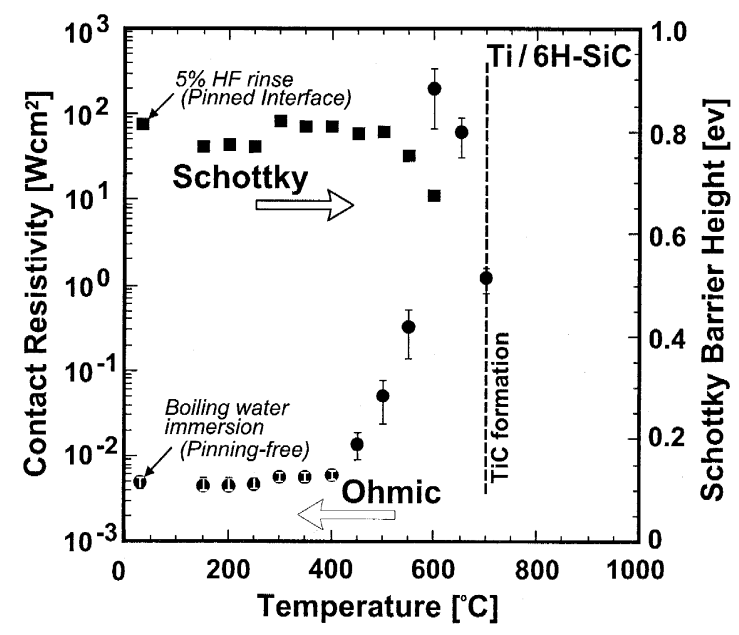

Fig. 3. Thermal stability of $\mathrm{Ti} / 6 \mathrm{H}-\mathrm{SiC}(0001)$ interfaces prepared by surface chemical treatments using boiling water and $5 \% \mathrm{HF}$ rinse.

interface chemical reaction by heat treatment?

We prepared pinning-controlled Ti ohmic contacts onto $6 \mathrm{H}-\mathrm{SiC}(0001)$ substrates formed by boiling water immersion and conventional Ti Schottky contacts onto the substrates formed by $5 \% \mathrm{HF}$ rinse. These two sets of samples are annealed in argon atmosphere. Ti electrodes were capped by $\mathrm{Ni}$ to prevent from surface oxidation. The ohmic contacts have no change in $\rho_{\mathrm{c}}$ until $400^{\circ} \mathrm{C}$ as shown in Fig. 3. Small widths of error bars suggest that $\mathrm{rc}$ is an essential value for the $\mathrm{Ti} / \mathrm{SiC}$ contacts. $\rho_{\mathrm{c}}$ increases drastically from $450^{\circ} \mathrm{C}$ and takes the maximum at $600^{\circ} \mathrm{C}$. It surprisingly turns into decrease after $600^{\circ} \mathrm{C}$. It was reported from transmission electron microscopy that epitaxial $\mathrm{TiC}$ forms at $700^{\circ} \mathrm{C}$ at the interface [14]. Since the ideal ohmic resistivities collapses in the regime between 450 to $600^{\circ} \mathrm{C}$, atoms at the interface start to break interface chemical bonds as an precedent phenomenon of interdiffusion, resulting in increase in $D_{\mathrm{it}}$. In the next regime from $650^{\circ} \mathrm{C}, D_{\mathrm{it}}$ decrease because of the recovery of interface ideality. On the other hand, the conventional Schottky contacts deteriorate from $550^{\circ} \mathrm{C}$. It is known that a rectifying property at the conventional $\mathrm{Ti} / \mathrm{SiC}$ interface recovers at $700^{\circ} \mathrm{C}$ for a longer anneal [14]. 


\section{Conclusions}

We proposed the first method to control Schottky barriers by changing the Fermi level pinning and by changing the metal work function. This method unifies Schottky barriers and ohmic conductivities in terms of their artificial control. It has a simple current transport mechanism with a minor effect of tunneling current. The pinning control was achieved by dipping silicon carbide crystals into $\mathrm{pH}$ modified buffered HF or boiling water before metallizations. These treatments have an effect to reduce the density of the surface states and the resultant density of the interface states. Ohmic interfaces were formed by selecting $\mathrm{Ti}$ with a low work function as a contact metal. Further, we found that Schottky barriers of Al contacts onto the $\mathrm{Si}(111)$ substrates with atomicallyflat surfaces prepared using cleaning by hot water and a slow oxidation technique by oxygen gas are lowered toward the ohmic regime. Moreover, we observe a precedent interdiffusion phenomenon breaking interface chemical bonds before forming an interfacial $\mathrm{TiC}$ layer at the $\mathrm{Ti} / 6 \mathrm{H}-\mathrm{SiC}(0001)$ interface.

\section{References}

[1] E.H. Rhoderick, R.H. Williams, Metal-Semiconductor Contacts, 2nd ed., Oxford University Press, Oxford, 1988, p. 20.

[2] S.M. Sze, Physics of Semiconductor Devices, 2nd ed., Ch. 5, Wiley, New York, 1981, p. 292.

[3] J.F. Fan, H. Oigawa, Y. Nannichi, Jpn. J. Appl. Phys. 27 (1988) L2125.

[4] W. Monch, Semiconductor Surfaces and Interfaces, 2nd ed., Springer Series in Surface Sciences 26, Springer-Verlag, Berlin, 1995.

[5] G.S. Higashi, Y.J. Chabal, G.W. Trucks, K. Raghavachari, Appl. Phys. Lett. 56 (1990) 656.

[6] S. Watanabe, M. Shigeno, N. Nakayama, T. Ito, Jpn. J. Appl. Phys. 12B (1991) 3575.

[7] K. Ohishi, T. Hattori, Jpn. J. Appl. Phys. 33 (1994) L675.

[8] S. Hara, T. Teraji, H. Okushi, K. Kajimura, Appl. Surf. Sci. 107 (1996) 218.

[9] G. Pensl, W.J. Choyke, Physica B 185 (1993) 264.

[10] S. Kurtin, T.C. McGill, C.A. Mead, Phys. Rev. Lett. 22 (1969) 1433.

[11] W. Kern, D.A. Puotinen, RCA Rev. 31 (1970) 187.

[12] F.G. Allen, G.W. Gobeli, Phys. Rev. 127 (1962) 150.

[13] H.B. Michaelson, J. Appl. Phys. 48 (1977) 4729.

[14] L.M. Porter, R.F. Davis, J.S. Bow, M.J. Kim, R.W. Carpenter, R.C. Glass, J. Mater. Res. 10 (1995) 668. 